\title{
Off resonance coupling between a cavity mode and an ensemble of driven spins
}

\author{
Hui Wang, Sergei Masis, Roei Levi, Oleg Shtempluk, and Eyal Buks \\ Andrew and Erna Viterbi Department of Electrical Engineering, Technion, Haifa 32000 Israel
}

(Dated: June 18, 2021)

\begin{abstract}
We study the interaction between a superconducting cavity and a spin ensemble. The response of a cavity mode is monitored while simultaneously the spins are driven at a frequency close to their Larmor frequency, which is tuned to a value much higher than the cavity resonance. We experimentally find that the effective damping rate of the cavity mode is shifted by the driven spins. The measured shift in the damping rate is attributed to the retarded response of the cavity mode to the driven spins. The experimental results are compared with theoretical predictions and fair agreement is found.
\end{abstract}

PACS numbers: 76.30.-v, 42.50.Pq

\section{INTRODUCTION}

Cavity quantum electrodynamics (CQED) [1] is the study of the interaction between matter and photons confined in a cavity. In the Jaynes-Cummings model [2] the matter is described using the two-level approximation, and only a single cavity mode is taken into account. The interaction has a relatively large effect on the cavity mode response when the ratio $E / \hbar \omega_{\mathrm{a}}$ between the energy gap $E$ separating the two levels and the cavity mode photon energy $\hbar \omega_{\mathrm{a}}$ is tuned close to unity. Recently, it was experimentally found that the cavity response exhibits higher order resonances in the nonlinear regime when the ratio $E / \hbar \omega_{\mathrm{a}}$ is tuned close to an integer value larger than unity [3].

In the current study we explore the case where $E / \hbar \omega_{\mathrm{a}} \gg 1[4]$. This is done by investigating the interaction between an ensemble of spins and a superconducting cavity mode 5 -7]. The energy separation between the spin energy eigenstates, which is given by $E=\hbar \omega_{\mathrm{L}}$, where $\omega_{\mathrm{L}}$ is the Larmor frequency, is tuned to a value much higher than the cavity mode photon energy $\hbar \omega_{\mathrm{a}}$. For this case the CQED interaction is expected to be negligibly small in the regime of weak driving. On the other hand, with an intense driving at an angular frequency close to $\omega_{\mathrm{L}}$ we observe a significant change in the cavity mode response.

In the current experiment the cavity mode effective damping rate is measured as a function of the spin driving amplitude and detuning frequency. The observed shift in the effective damping rate is attributed to the retarded response of the cavity mode to the driven spins. Related effects of Sisyphus cooling, amplification, lasing and selfexcited oscillation have been theoretically predicted in other systems having a similar retarded response [8 8 - 2 .

\section{EXPERIMENT}

Significant change in the response of the measured cavity mode of angular frequency $\omega_{\mathrm{a}}$ is possible only when intense driving is applied to the spins. In order to allow sufficiently strong driving, the spin ensemble is coupled

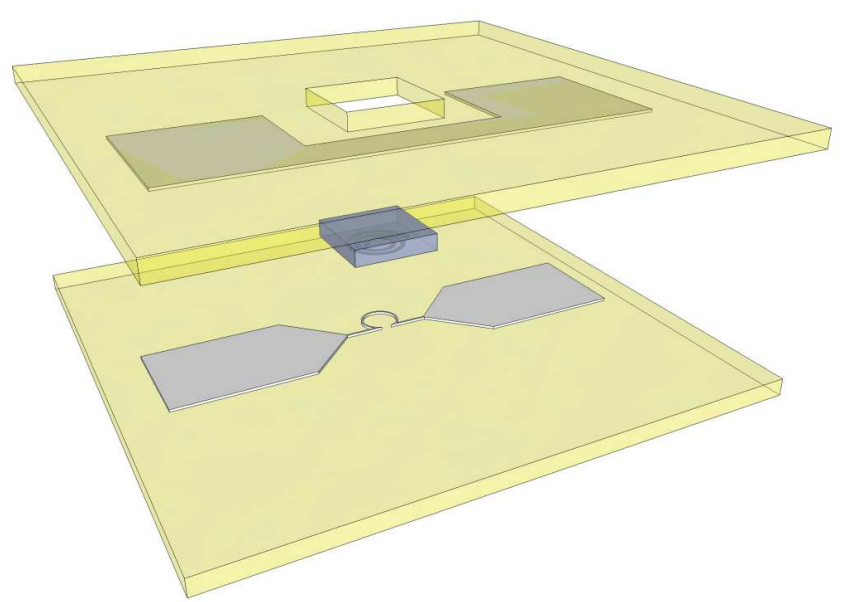

FIG. 1: The device is made of two $40 \mathrm{~mm} \times 40 \mathrm{~mm} \times 0.5 \mathrm{~mm}$ sapphire wafers carrying the radio frequency omega resonator, and a $5 \mathrm{~mm} \times 5 \mathrm{~mm} \times 0.5 \mathrm{~mm}$ silicon wafer carrying the microwave spiral resonator. The DPPH powder is placed between the omega inductor and the spiral. The 3 wafers are vertically shifted in the sketch for clarity. In the assembled device both the top sapphire wafer and the silicon wafer are placed directly on top of the bottom sapphire wafer. The three wafers and a loop antenna are assembled together inside a package made of high conductivity oxygen free copper. Both omega and spiral resonators are made by DC-magnetron sputtering of a $200 \mathrm{~nm}$ thick niobium layer. The radius of the omega inductor is $500 \mu \mathrm{m}$ and the linewidth is $40 \mu \mathrm{m}$. The spiral dimensions are: inner radius $500 \mu \mathrm{m}$, outer radius $580 \mu \mathrm{m}$, linewidth $10 \mu \mathrm{m}$ and number of turns 4 . The measured frequency of the omega (spiral) resonator is $\omega_{\mathrm{a}} / 2 \pi=0.173 \mathrm{GHz}$ $\left(\omega_{\mathrm{b}} / 2 \pi=2.00 \mathrm{GHz}\right)$, whereas the value obtained from numerically simulating the structure is $0.176 \mathrm{GHz}(2.07 \mathrm{GHz})$.

to an additional cavity mode having angular frequency $\omega_{\mathrm{b}} \gg \omega_{\mathrm{a}}$. When the Larmor frequency $\omega_{\mathrm{L}}$ is tuned to a value close to $\omega_{\mathrm{b}}$, the additional cavity mode allows enhancing the spin driving amplitude.

A sketch of the device is seen in Fig. 1 It is made of two sapphire wafers and a high resistivity silicon wafer that are attached together to form a dual band resonator. A radio frequency resonator of angular frequency $\omega_{\mathrm{a}}$ is 


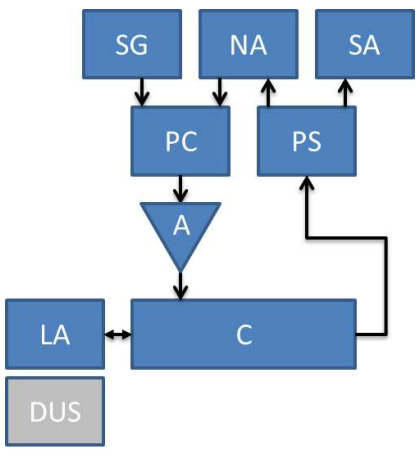

FIG. 2: The experimental setup. A power combiner (PC) is employed for combining the injected signals of a signal generator (SG) and a network analyzer (NA). The combined injected signal is transmitted through an amplifier (A) and a coupler (C), and feeds the loop antenna (LA), which is positioned above the device under study (DUS). The back-reflected signal is splitted by a power splitter (PS) and measured by both a NA and a spectrum analyzer (SA).

constructed by integrating an inductor in the shape of the greek letter $\Omega$ [13] made on the bottom sapphire wafer, and two capacitors in series, which are formed between the two sapphire wafers. A square hole is made in the upper sapphire wafer in order to allow inserting the silicon wafer, which carries a spiral shaped microwave resonator having angular frequency $\omega_{\mathrm{b}}$ [14, 15].

Both the resonators are designed to be efficiently coupled to the spin ensemble of diphenylpicrylhydrazyl (DPPH) powder, placed in between them. This radical, which contains three benzene rings, has a single unpaired electron, which gives rise to Landé g-factor of 2.0036 [16, 17]. A sketch of the experimental setup is seen in Fig. 2. A loop antenna is employed for delivering input and output signals to both resonators.

The measured reflectivity near the electron spin resonance (ESR) of the omega and spiral resonators is seen in Fig. 3 (a) and (b), respectively [18]. Fitting the data with theory [e.g. Eq. (4) of Ref. [3]] allows extracting the value of the coupling coefficient $g_{\mathrm{a}}\left(g_{\mathrm{b}}\right)$, which characterizes the interaction between the spin ensemble and the omega (spiral) resonator, and which is found to be $g_{\mathrm{a}}=13 \mathrm{MHz}\left(g_{\mathrm{b}}=83 \mathrm{MHz}\right)$.

The linear response of the decoupled omega resonator is characterized by a complex angular frequency given by $\omega_{\mathrm{a}}-i \gamma_{\mathrm{a}}$, where $\gamma_{\mathrm{a}}$ is the mode damping rate. The effect of coupling on the linear response of the mode can be described in terms of an effective complex angular frequency $\Omega_{\mathrm{a}}=\omega_{\mathrm{a}}-i \gamma_{\mathrm{a}}+\Upsilon_{\mathrm{a}}$, where $\Upsilon_{\mathrm{a}}$ represents the coupling induced frequency shift. The complex angular frequency $\Omega_{\mathrm{a}}$ can be extracted from the lineshape of the measured cavity reflectivity vs. frequency curves. The change in the damping rate $-\operatorname{Im} \Upsilon_{\mathrm{a}}$ is seen in the colorcoded plots of Fig. 4 as a function of the Larmor frequency $\omega_{\mathrm{L}}$ and the spin driving angular frequency $\omega_{\mathrm{p}}$.
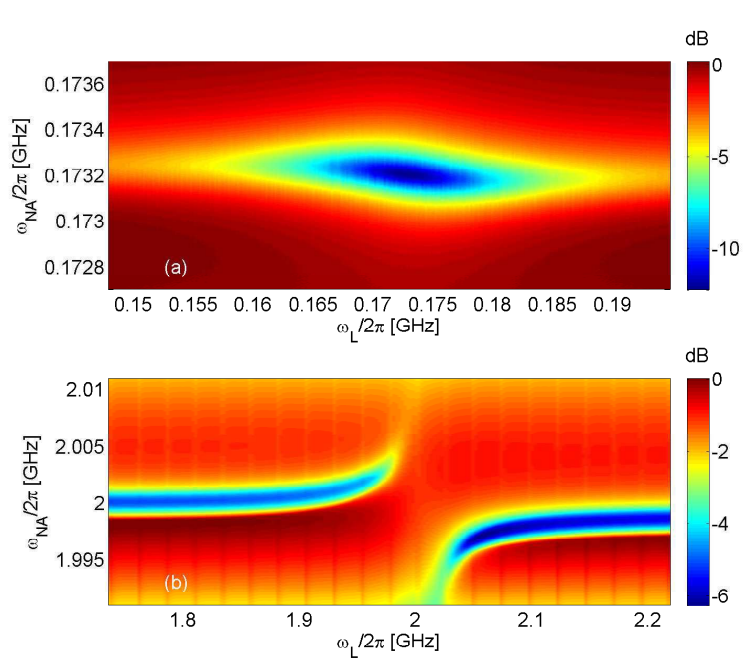

FIG. 3: ESR of (a) the omega and (b) the spiral resonators. The color coded plots display the measured reflectivity coefficient $\left|S_{11}\right|^{2}$ vs. $\omega_{\mathrm{L}}$ (i.e. vs. static magnetic field) and the probing frequency $\omega_{\mathrm{NA}}$. Measurements are performed by a network analyzer at a temperature of $T=3.1 \mathrm{~K}$, for which the polarization coefficient $p_{0}$ [see Eq. A20 ] is given by $p_{0}=-1.4 \times 10^{-3}\left(p_{0}=-1.6 \times 10^{-2}\right)$ for the omega (spiral) resonator.
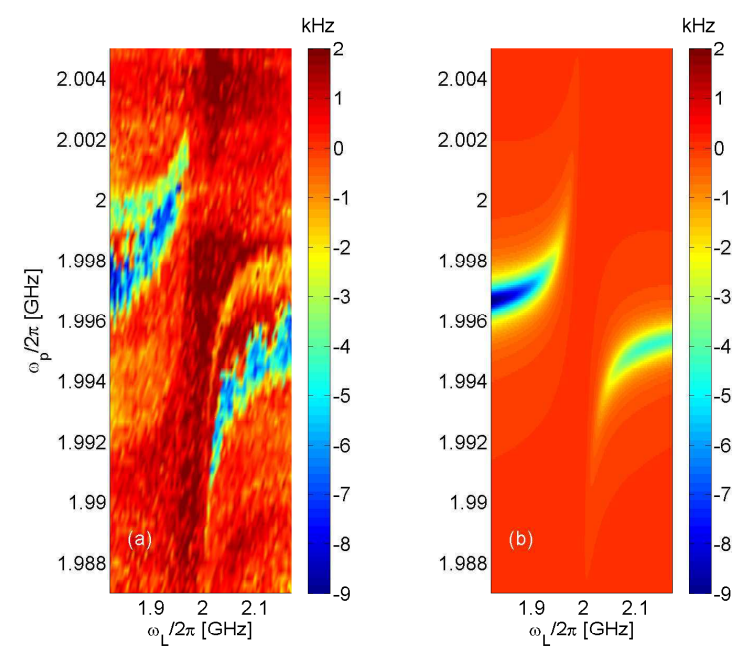

FIG. 4: The measured (a) and calculated (b) change in the damping rate $-\operatorname{Im} \Upsilon_{a}$ vs. $\omega_{L}$ and the pump frequency $\omega_{\mathrm{p}}$. The experimental value is obtained from the lineshape of the omega resonance. The Larmor frequency $\omega_{\mathrm{L}}$ is tuned by applying a static magnetic field in a direction parallel to the wafers. The pump power is set to the value $17 \mathrm{dBm}$, which corresponds to a driving amplitude of $\omega_{1} / 2 \pi=12 \mathrm{MHz}$. The calculated shift (b) is based on Eqs. (1) and (2). The following parameters are used in the calculation $\gamma_{\mathrm{b}}=0.4 \mathrm{MHz}$ and $\gamma_{2}=8.3 \mathrm{MHz}$ (other parameters are specified above). 


\section{THEORY}

To account for the experimental findings, two possible contributions to $\Upsilon_{\mathrm{a}}$, which is expressed as $\Upsilon_{\mathrm{a}}=$ $\Upsilon_{\mathrm{aL}}+\Upsilon_{\mathrm{ab}}$, have been theoretically estimated. While $\Upsilon_{\mathrm{aL}}$ represents the shift induced by the coupling to the driven spins, the $\Upsilon_{\mathrm{ab}}$ contribution originates from the coupling to the driven spiral mode.

A magnetic field having two mutually orthogonal components, a static component and an alternating one at an angular frequency $\omega_{\mathrm{p}}$, is applied to the spin ensemble. The amplitude of the static (alternating) component is $\gamma_{\mathrm{g}}^{-1} \omega_{\mathrm{L}}\left(\gamma_{\mathrm{g}}^{-1} \omega_{1}\right)$, where $\gamma_{\mathrm{g}}$ is the electron spin gyromagnetic ratio. The frequency shift $\Upsilon_{\mathrm{aL}}$ is found to be given by [see appendix A and Eq. (A46]

$$
\Upsilon_{\mathrm{aL}}=\frac{\frac{\frac{8 g_{\mathrm{a}}^{2} \omega_{1}^{2}}{\omega_{\mathrm{a}}^{2} \gamma_{2}} \frac{\Delta_{\mathrm{pL}}}{\gamma_{2}}\left(i-\frac{2 \gamma_{2}}{\omega_{\mathrm{a}}}\right)}{1+\frac{\Delta_{\mathrm{pL}}^{2}}{\gamma_{2}^{2}}+\frac{4 \omega_{1}^{2}}{\gamma_{1} \gamma_{2}}} p_{0}}{\frac{\gamma_{1}}{\omega_{\mathrm{a}}}\left(\frac{\omega_{\mathrm{R}}^{2}+\eta \omega_{\mathrm{a}}^{2}}{\omega_{\mathrm{a}}^{2}}-1\right)-i\left(\frac{\omega_{\mathrm{R}}^{2}}{\omega_{\mathrm{a}}^{2}}-1\right)},
$$

where $\Delta_{\mathrm{pL}}=\omega_{\mathrm{p}}-\omega_{\mathrm{L}}$ is the detuning, $\gamma_{1}\left(\gamma_{2}\right)$ is the longitudinal (transverse) spin relaxation rate, $p_{0}$ is the spin polarization in thermal equilibrium [see Eq. [A20] $], \omega_{\mathrm{R}}=$ $\sqrt{4 \omega_{1}^{2}+\Delta_{\mathrm{pL}}^{2}}$ is the Rabi frequency of the driven spins and $\eta$ is given by $\eta=\left(2 \gamma_{2} / \gamma_{1}\right)\left[2 \omega_{1}^{2}\left(1-\gamma_{1} / \gamma_{2}\right) / \omega_{\mathrm{a}}^{2}-1\right]$ [see Eq. (A47)]. Note that Eq. (11) is obtained by assuming that $\left|\Delta_{\mathrm{pL}}\right| \ll \omega_{\mathrm{L}}, \gamma_{\mathrm{a}} \ll \omega_{\mathrm{a}}$ and $\gamma_{1}, \gamma_{2} \ll \omega_{\mathrm{a}}$.

The real part of $\Upsilon_{\mathrm{aL}}$ is the cavity mode angular frequency change, induced by the coupling to the driven spins. The imaginary part of $\Upsilon_{\mathrm{aL}}$ is related to the induced damping rate change $\gamma_{\mathrm{aL}}$ by $\gamma_{\mathrm{aL}}=-\operatorname{Im} \Upsilon_{\mathrm{aL}}$. The color coded plot in Fig. 5(a) exhibits the dependence of the normalized change in damping rate $\gamma_{\mathrm{a}} / \omega_{\mathrm{a}}$ on the normalized detuning $\Delta_{\mathrm{pL}} / \omega_{\mathrm{a}}$ and the normalized driving amplitude $\omega_{1} / \omega_{\mathrm{a}}$. When the driving is red detuned, i.e. when $\Delta_{\mathrm{pL}}$ is negative, the change in damping rate $\gamma_{\mathrm{aL}}$ is positive, and consequently mode cooling is expected to occur [19]. The opposite behavior occurs with blue detuning, i.e. when $\Delta_{\mathrm{pL}}$ is positive. For both cases, large change in the effective cavity mode damping rate occurs near the overlaid dotted line in Fig. 5)(a), along which the Rabi frequency $\omega_{R}$ coincides with the cavity mode frequency $\omega_{\mathrm{a}}$, i.e. $\Delta_{\mathrm{pL}}= \pm \sqrt{\omega_{\mathrm{a}}^{2}-4 \omega_{1}^{2}}$. This behavior can be attributed to the fact that along the dotted line, i.e. when $\omega_{\mathrm{R}}=\omega_{\mathrm{a}}$, the imaginary part of the denominator of Eq. (1) vanishes, and consequently $\left|\Upsilon_{\mathrm{aL}}\right|$ peaks. The largest change in damping rate, which is denoted by $\gamma_{\mathrm{aL}, \mathrm{max}}$, can be evaluated by analyzing the expression given by Eq. (11). In the absence of spin dephasing, i.e. when $\gamma_{1} / \gamma_{2}=2$, it is found that the largest change, which is given by $\gamma_{\mathrm{aL}, \max } \simeq 0.437 \times g_{\mathrm{a}}^{2} p_{0} / \gamma_{2}$, occurs at the points $\left(\Delta_{\mathrm{pL}} / \omega_{\mathrm{a}}, \omega_{1} / \omega_{\mathrm{a}}\right) \simeq( \pm 0.527,0.425)$, which are labeled by crosses in Fig. 5(a). In the current experiment, however, these points are not accessible since $\omega_{1} \ll \omega_{\text {a }}$.

The underlying mechanism responsible for the change in the effective cavity mode damping rate that is induced
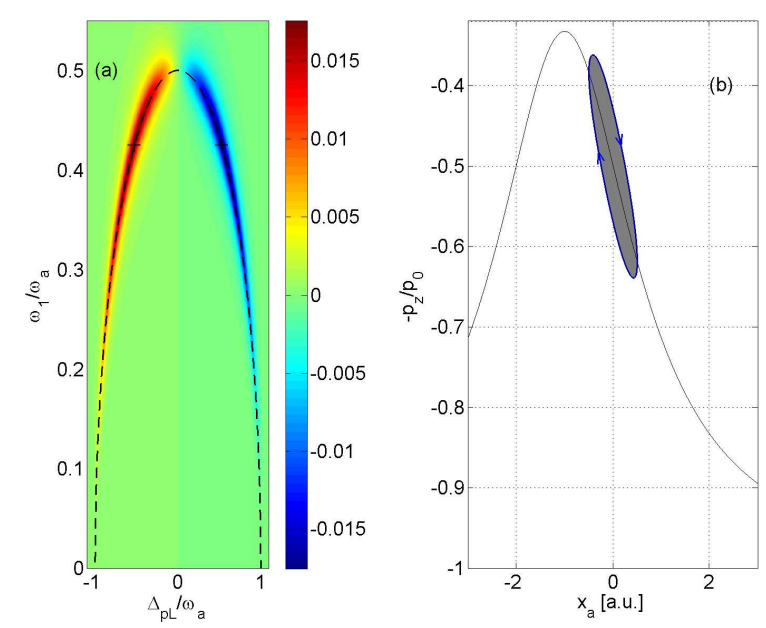

FIG. 5: The contribution to cavity mode damping rate $\gamma_{\mathrm{aL}}$ due to coupling to the driven spins. (a) The normalized contribution $\gamma_{\mathrm{aL}} / \omega_{\mathrm{a}}$ vs. normalized detuning $\Delta_{\mathrm{pL}} / \omega_{\mathrm{a}}$ and normalized driving amplitude $\omega_{1} / \omega_{\mathrm{a}}$. The calculation is based on Eq. (1) with the following assumed parameters $\gamma_{1} / \omega_{\mathrm{a}}=2 \gamma_{2} / \omega_{\mathrm{a}}=0.05, g_{\mathrm{a}} / \omega_{\mathrm{a}}=0.1$ and $p_{0}=-0.1$. (b) Normalized spin polarization $-p_{z} / p_{0}$ vs. cavity mode amplitude $x_{\mathrm{a}}$ for the case of blue detuned driving. The black solid line represents the steady state normalized spin polarization $-p_{z 0} / p_{0}$. Retardation in the response of the spins to periodic oscillation of $x_{\mathrm{a}}$ is illustrated by the blue closed curved.

by the coupling to the driven spins can be described as follows [19]. As can be seen from the equation of motion for the cavity mode (A11), the coupling gives rise to a forcing term acting on the cavity mode, which is proportional to the spin polarization that is denoted by $p_{z}$. On the other hand, as can be seen from Eq. A12 below, the same coupling effectively shifts the Larmor frequency of the spins, and consequently the effective spin driving detuning, which is given by $\Delta_{\mathrm{pL} \text {,eff }}=\Delta_{\mathrm{pL}}+g_{\mathrm{a}} x_{\mathrm{a}}$, becomes dependent on the cavity mode amplitude $x_{\mathrm{a}}$ [see Eq. A25)]. For any fixed value of the cavity mode amplitude $x_{\mathrm{a}}$ the spin polarization $p_{z}$ in steady state, which is denoted by $p_{z 0}$, can be calculated using Eq. A43) below. The dependence of $p_{z 0}$ on $x_{\mathrm{a}}$ is demonstrated by the solid black line in Fig. 5(b) for the case of blue detuned spin driving. Consider first the adiabatic case, for which it is assumed that $\omega_{\mathrm{a}} \ll \gamma_{1,2}$. For this case the dynamics of the cavity mode is assumed to be relatively slow, and consequently the spin polarization $p_{z}$ is expected to remain very close to the steady state value given by $p_{z 0}$, i.e. to adiabatically follow the $x_{\mathrm{a}}$ dependent instantaneous steady state value. Therefore, no change in the cavity mode damping rate is expected in the adiabatic limit. This behavior is consistent with the fact that $\operatorname{Im} \Upsilon_{\mathrm{aL}} \rightarrow 0$ in the limit $\omega_{\mathrm{a}} / \gamma_{1,2} \rightarrow 0$. Note, however, that the expression given by Eq. (1) is not valid in the adiabatic limit. 
Large deviation between the momentary polarization $p_{z}$ and the steady state value $p_{z 0}$ is possible in the nonadiabatic case, where the response of the spins to the time evolution of the cavity mode becomes retarded. The closed curve in Fig. [5) represents the periodic time evolution of $p_{z}$ for the case where the cavity mode oscillates at a fixed amplitude at its resonance frequency around the point $x_{\mathrm{a}}=0$. Since $p_{z}$ is proportional to the force acting on the cavity mode, the area colored in gray in Fig. 5 (b) is proportional to the net work done on the cavity mode per cycle. While the area is positive for the case of blue detuning, which is demonstrated by Fig. 5(b), red detuning gives rise to negative values, i.e. to energy flow away from the cavity mode. These affects of energy flow between the cavity mode and the driven spins give rise to the above discussed change in the effective cavity mode damping rate.

The frequency shift due to the driven spiral mode is attributed to an intermode coupling term in the Hamiltonian of the coupled system, which is assumed to be given by $K\left(A_{\mathrm{a}}+A_{\mathrm{a}}^{\dagger}\right)\left(A_{\mathrm{b}}+A_{\mathrm{b}}^{\dagger}\right)^{2}$, where $A_{\mathrm{a}}\left(A_{\mathrm{b}}\right)$ is an annihilation operator of the omega (spiral) resonator, and $K$ is the intermode coupling coefficient [see Eq.(B1)]. The contribution $\Upsilon_{\mathrm{ab}}$ is found to be given by [see appendix $\mathrm{B}$ and Eqs. (B19) and (B30)]

$$
\begin{aligned}
\Upsilon_{\mathrm{ab}} & =\frac{4 K^{2}\left|F_{\mathrm{bf}}\right|^{2}}{\omega_{\mathrm{D}}^{2}+\gamma_{\mathrm{b}}^{2}} \\
& \left(\frac{\frac{\omega_{\mathrm{D}}}{\gamma_{\mathrm{b}}^{2}}}{\left(\frac{i\left(\omega_{\mathrm{a}}-\omega_{\mathrm{D}}\right)}{\gamma_{\mathrm{b}}}-1\right)\left(\frac{i\left(\omega_{\mathrm{a}}+\omega_{\mathrm{D}}\right)}{\gamma_{\mathrm{b}}}-1\right)}+\frac{1+\frac{i \gamma_{\mathrm{b}}}{\omega_{\mathrm{s}}}}{\omega_{\mathrm{s}}}\right),
\end{aligned}
$$

where $F_{\mathrm{bf}}$ and $\omega_{\mathrm{D}}$ are the amplitude and angular frequency detuning, respectively, of the spiral mode driving, $\omega_{\mathrm{b}}$ and $\gamma_{\mathrm{b}}$ are the spiral mode angular frequency and damping rate, respectively, and $\omega_{\mathrm{s}}=2 \omega_{\mathrm{b}}-\omega_{\mathrm{a}}$. Note that when $\gamma_{\mathrm{b}} \ll \omega_{\mathrm{a}}$ and $\gamma_{\mathrm{b}} \ll \omega_{\mathrm{s}}$, the first term in the second row of Eq. (22) becomes negligibly small provided that $\left|\omega_{\mathrm{D}}\right| \ll \omega_{\mathrm{a}}^{2} / \omega_{\mathrm{s}}$.

\section{DISCUSSION}

As can be seen from the comparison between Fig. 4 (a) and Fig. 4(b), fair agreement is obtained between data and theory. Reasonable agreement cannot be obtained unless both contributions $\Upsilon_{\mathrm{aL}}$ [Eq. (1)] and $\Upsilon_{\mathrm{ab}}$ [Eq. (2)] are taken into account. The contribution of $\Upsilon_{\mathrm{ab}}$ is dominated by the second term in the second row of Eq. (2).

Our results demonstrate the ability to modify the effective damping rate of a cavity mode by driving spins that are coupled to the mode. Red detuned driving provides a positive contribution to the damping rate, whereas negative contribution can be obtained by blue detuned driving. For the former case this effect can be utilized for cooling down a cavity mode, while the later case of blue detuning may allow the self excitation of oscillation. Operating close to the threshold of self-excited oscillation, i.e. close to the point where the total effective damping vanishes, may be useful for some sensing applications, since the system is expected to become highly responsive to external perturbations near the threshold.

As was shown above, relatively large change in the damping rate can be induced provided that the Rabi frequency $\omega_{\mathrm{R}}$ of the driven spins becomes comparable to the cavity mode frequency $\omega_{\mathrm{a}}$ (see Fig. [5). Unfortunately, this region is inaccessible with the devices that have been investigated in the current experiment. However, in other CQED systems the condition $\omega_{\mathrm{R}} \simeq \omega_{\mathrm{a}}$ can be more easily satisfied. For example, with superconducting CQED systems both strong 20 23] and ultra-strong [24, 25] coupling is possible. This together with the ability to drive a Josephson qubit with Rabi frequencies high in the radio frequency band, may allow satisfying the condition $\omega_{\mathrm{R}} \simeq \omega_{\mathrm{a}}$ with a strongly coupled cavity mode. As was shown above, a large change in cavity mode damping rate, on the order of $g_{\mathrm{a}}^{2}\left|p_{0}\right| / \gamma_{2}$, is possible provided that the region where $\omega_{\mathrm{R}} \simeq \omega_{\mathrm{a}}$ becomes accessible. For a typical superconducting CQED system, the damping rate of a decoupled cavity mode is far smaller than $g_{\mathrm{a}}^{2}\left|p_{0}\right| / \gamma_{2}$, and thus reaching this region may allow efficiently cooling down cavity modes by off-resonance qubit driving.

This work was supported by the Israel Science Foundation, the bi-national science foundation, the Security Research Foundation in the Technion and the Russell Berrie Nanotechnology Institute. HW acknowledges support by the Shatz fellowships and by the Viterbi fellowships.

\section{Appendix A: Coupling to driven spins}

Consider an ensemble of spin $1 / 2$ particles coupled to a cavity mode. The ensemble is characterized by a longitudinal (spin-lattice) relaxation rate $\gamma_{1}$ and by a transverse (spin-spin) relaxation rate $\gamma_{2}$. An external magnetic field is applied, having a component alternating with angular frequency $\omega_{\mathrm{p}}$, and an orthogonal static component. The amplitude of the alternating (static) component is $\gamma_{\mathrm{g}}^{-1} \omega_{1}\left(\gamma_{\mathrm{g}}^{-1} \omega_{\mathrm{L}}\right)$, where $\gamma_{\mathrm{g}}=2 \pi \times 28.03 \mathrm{GHz} \mathrm{T}^{-1}$ is the electron spin gyromagnetic ratio. It is assumed that driving is applied close to the electron spin resonance, i.e. $\left|\Delta_{\mathrm{pL}}\right| \ll \omega_{\mathrm{L}}$, where $\Delta_{\mathrm{pL}}=\omega_{\mathrm{p}}-\omega_{\mathrm{L}}$ is the detuning. The cavity mode is characterized by an angular frequency $\omega_{\mathrm{a}}$ and a damping rate $\gamma_{\mathrm{a}}$. The coupling between the cavity mode and the spin ensemble is characterized by a longitudinal coupling coefficient $g_{\mathrm{a}}$. 


\section{Equations of motion}

The Hamiltonian of the closed system is taken to be given by

$$
\begin{aligned}
\hbar^{-1} \mathcal{H}_{\mathrm{aL}} & =\omega_{\mathrm{a}}\left(A_{\mathrm{a}}^{\dagger} A_{\mathrm{a}}+\frac{1}{2}\right)+\frac{\omega_{\mathrm{L}}}{2} \Sigma_{z} \\
& +\omega_{1}\left(e^{-i \omega_{\mathrm{p}} t} \Sigma_{+}+e^{i \omega_{\mathrm{p}} t} \Sigma_{-}\right) \\
& -g_{\mathrm{a}}\left(A_{\mathrm{a}}+A_{\mathrm{a}}^{\dagger}\right) \Sigma_{z}
\end{aligned}
$$

The Heisenberg equations of motion are generated according to

$$
\frac{\mathrm{d} O}{\mathrm{~d} t}=-i\left[O, \hbar^{-1} \mathcal{H}_{\mathrm{aL}}\right]
$$

where $O$ is an operator. Using the commutation relations

$$
\begin{aligned}
{\left[A_{\mathrm{a}}, A_{\mathrm{a}}^{\dagger}\right] } & =1, \\
{\left[\Sigma_{z}, \Sigma_{+}\right] } & =2 \Sigma_{+}, \\
{\left[\Sigma_{z}, \Sigma_{-}\right] } & =-2 \Sigma_{-}, \\
{\left[\Sigma_{+}, \Sigma_{-}\right] } & =\Sigma_{z},
\end{aligned}
$$

one obtains

$$
\begin{gathered}
\frac{\mathrm{d} A_{\mathrm{a}}}{\mathrm{d} t}+i \omega_{\mathrm{a}} A_{\mathrm{a}}-i g_{\mathrm{a}} \Sigma_{z}=0, \\
\frac{\mathrm{d} \Sigma_{+}}{\mathrm{d} t}-i \Omega_{\mathrm{L}} \Sigma_{+}+i \omega_{1}^{\dagger} e^{i \omega_{\mathrm{p}} t} \Sigma_{z}=0,
\end{gathered}
$$

and

$$
\frac{\mathrm{d} \Sigma_{z}}{\mathrm{~d} t}+2 i\left(\Sigma_{+} \omega_{1} e^{-i \omega_{\mathrm{p}} t}-\Sigma_{-} \omega_{1}^{\dagger} e^{i \omega_{\mathrm{p}} t}\right)=0
$$

where

$$
\Omega_{\mathrm{L}}=\omega_{\mathrm{L}}-2 g_{\mathrm{a}}\left(A_{\mathrm{a}}+A_{\mathrm{a}}^{\dagger}\right)
$$

In the next step damping is introduced, and the resultant equations for the operators $A_{\mathrm{a}}, \Sigma_{+}$and $\Sigma_{z}$ are thermally averaged. This procedure leads to

$$
\begin{gathered}
\frac{\mathrm{d} a}{\mathrm{~d} t}+\Theta_{\mathrm{a}}=0, \\
\frac{\mathrm{d} p_{+}}{\mathrm{d} t}+\Theta_{+}=0, \\
\frac{\mathrm{d} p_{z}}{\mathrm{~d} t}+\Theta_{z}=0,
\end{gathered}
$$

where

$$
\begin{aligned}
a & =\left\langle A_{\mathrm{a}}\right\rangle, \\
p_{+} & =e^{-i \omega_{\mathrm{p}} t}\left\langle\Sigma_{+}\right\rangle, \\
p_{z} & =\left\langle\Sigma_{z}\right\rangle=p_{z},
\end{aligned}
$$

triangle brackets denote thermal averaging, the functions $\Theta_{\mathrm{a}}, \Theta_{+}$and $\Theta_{z}$ are given by

$$
\begin{gathered}
\Theta_{\mathrm{a}}=\lambda_{\mathrm{a}} a-i g_{\mathrm{a}} p_{z}, \\
\Theta_{+}=\left(i \Delta_{\mathrm{pL}}+\gamma_{2}\right) p_{+} \\
+i \omega_{1} p_{z}+2 i g_{\mathrm{a}}\left(a+a^{*}\right) p_{+},
\end{gathered}
$$$$
\Theta_{z}=\gamma_{1}\left(p_{z}-p_{0}\right)+2 i \omega_{1}\left(p_{+}-p_{+}^{*}\right)
$$

the cavity eigenvalue $\lambda_{\mathrm{a}}$ is given by $\lambda_{\mathrm{a}}=i \omega_{\mathrm{a}}+\gamma_{\mathrm{a}}$, the coefficient

$$
p_{0}=-\tanh \left(\frac{\hbar \omega_{\mathrm{L}}}{2 k_{\mathrm{B}} T}\right)
$$

is the value of $p_{z}$ in thermal equilibrium in the absence of both driving and coupling, $k_{\mathrm{B}}$ is the Boltzmann's constant and $T$ is the temperature.

\section{The cavity eigenvalue}

The $5 \times 5$ Jacobian matrix

$$
J=\frac{\partial\left(\Theta_{\mathrm{a}}, \Theta_{\mathrm{a}}^{*}, \Theta_{+}, \Theta_{+}^{*}, \Theta_{z}\right)}{\partial\left(a, a^{*}, p_{+}, p_{+}^{*}, p_{z}\right)},
$$

can be expressed as $J=J_{0}+g_{\mathrm{a}} V$, where the matrix $J_{0}$ in a block form is given by

$$
J_{0}=\left(\begin{array}{cc|c}
\lambda_{\mathrm{a}} & 0 & 0 \\
0 & \lambda_{\mathrm{a}}^{*} & 0 \\
\hline 0 & J_{\mathrm{L}}
\end{array}\right)
$$

the block $J_{\mathrm{L}}$ is given by

$$
J_{\mathrm{L}}=\left(\begin{array}{ccc}
i \Delta_{\mathrm{pL}}+\gamma_{2} & 0 & i \omega_{1} \\
0 & -i \Delta_{\mathrm{pL}}+\gamma_{2} & -i \omega_{1} \\
2 i \omega_{1} & -2 i \omega_{1} & \gamma_{1}
\end{array}\right)
$$

the matrix $V$ is given by

$$
V=\left(\begin{array}{ccccc}
0 & 0 & 0 & 0 & -i \\
0 & 0 & 0 & 0 & i \\
2 i p_{+} & 2 i p_{+} & i x_{\mathrm{a}} & 0 & 0 \\
-2 i p_{+}^{*} & -2 i p_{+}^{*} & 0 & -i x_{\mathrm{a}} & 0 \\
0 & 0 & 0 & 0 & 0
\end{array}\right)
$$

and

$$
x_{\mathrm{a}}=2\left(a+a^{*}\right) \text {. }
$$

Let $\lambda_{1}, \lambda_{2}, \cdots, \lambda_{5}$ be the five eigenvalues of $J=J_{0}+$ $g_{\mathrm{a}} V$. In the limit $g_{\mathrm{a}} \rightarrow 0$, i.e. when the cavity mode is decoupled from the spins, it is assumed that $\lambda_{1} \rightarrow \lambda_{\mathrm{a}}$. 
When $g_{\mathrm{a}}$ is sufficiently small the eigenvalue $\lambda_{1}$, which henceforth is referred to as the cavity eigenvalue, can be calculated using perturbation theory. For the case of high quality factor (i.e. the case where $\gamma_{\mathrm{a}} \ll \omega_{\mathrm{a}}$ ) $\lambda_{1}$ is found to be given to second order in $g_{\mathrm{a}}$ by

$$
\lambda_{1}=i \omega_{\mathrm{a}}+\gamma_{\mathrm{a}}+g_{\mathrm{a}} V_{11}-g_{\mathrm{a}}^{2}\left(V R\left(\omega_{\mathrm{a}}\right) V\right)_{11}+O\left(g_{\mathrm{a}}^{3}\right),
$$

where the $5 \times 5$ matrix $R(\omega)$ in a block form is given by

$$
R\left(\omega^{\prime}\right)=\left(\begin{array}{cc|c}
0 & 0 & 0 \\
0 & 0 & 0 \\
\hline 0 & \chi_{\mathrm{L}}\left(\omega^{\prime}\right)
\end{array}\right),
$$

where the $3 \times 3$ spin susceptibility matrix $\chi_{\mathrm{L}}\left(\omega^{\prime}\right)$ is given by

$$
\chi_{\mathrm{L}}\left(\omega^{\prime}\right)=\left(J_{\mathrm{L}}-i \omega^{\prime}\right)^{-1} .
$$

With the help of Eq. (A24) one finds that

$$
\lambda_{1}=i \omega_{\mathrm{a}}+\gamma_{\mathrm{a}}+\Lambda_{1}+O\left(g_{\mathrm{a}}^{3}\right),
$$

where

$$
\Lambda_{1}=2 g_{\mathrm{a}}^{2}\left[p_{+}^{*}\left(\chi_{\mathrm{L}}\left(\omega_{\mathrm{a}}\right)\right)_{32}-p_{+}\left(\chi_{\mathrm{L}}\left(\omega_{\mathrm{a}}\right)\right)_{31}\right] .
$$

The following holds [see Eq. A23]

$$
=\frac{1}{D_{\mathrm{L}}}\left(\begin{array}{ccc}
\chi_{\mathrm{L}}\left(\omega_{\mathrm{a}}\right) & \\
D_{3}+2 \omega_{1}^{2} & 2 \omega_{1}^{2} & -i \omega_{1} D_{2} \\
2 \omega_{1}^{2} & D_{1} D_{3}+2 \omega_{1}^{2} & i \omega_{1} D_{1} \\
-2 i \omega_{1} D_{2} & 2 i \omega_{1} D_{1} & D_{1} D_{2}
\end{array}\right),
$$

where

$$
\begin{aligned}
& D_{1}=i \Delta_{\mathrm{pL}}+\gamma_{2}-i \omega_{\mathrm{a}} \\
& D_{2}=-i \Delta_{\mathrm{pL}}+\gamma_{2}-i \omega_{\mathrm{a}} \\
& D_{3}=\gamma_{1}-i \omega_{\mathrm{a}} \\
& D_{\mathrm{L}}=D_{1} D_{2} D_{3}+2 \omega_{1}^{2}\left(D_{1}+D_{2}\right) .
\end{aligned}
$$

The determinant $D_{\mathrm{L}}$ can be expressed as [see Eq. A35)]

$$
\frac{D_{\mathrm{L}}}{\omega_{\mathrm{a}}^{3}}=\frac{\gamma_{1}}{\omega_{\mathrm{a}}}\left(\frac{\Delta_{\mathrm{pL}}^{2}-\omega_{\mathrm{dR}}^{2}}{\omega_{\mathrm{a}}^{2}}\right)-i\left(\frac{\Delta_{\mathrm{pL}}^{2}-\omega_{\mathrm{dI}}^{2}}{\omega_{\mathrm{a}}^{2}}\right),
$$

where

$$
\frac{\omega_{\mathrm{dR}}}{\omega_{\mathrm{a}}}=\sqrt{1+\frac{2 \gamma_{2}}{\gamma_{1}}\left(1-\frac{2 \omega_{1}^{2}}{\omega_{\mathrm{a}}^{2}}\right)-\frac{\gamma_{2}^{2}}{\omega_{\mathrm{a}}^{2}}},
$$

and

$$
\frac{\omega_{\mathrm{dI}}}{\omega_{\mathrm{a}}}=\sqrt{1-\frac{4 \omega_{1}^{2}}{\omega_{\mathrm{a}}^{2}}-\frac{\left(2 \gamma_{1}+\gamma_{2}\right) \gamma_{2}}{\omega_{\mathrm{a}}^{2}}}
$$

Using these notations Eq. (A30) becomes

$$
\frac{\Lambda_{1}}{\omega_{\mathrm{a}}}=\frac{8 g_{\mathrm{a}}^{2} \omega_{1}}{\omega_{\mathrm{a}}^{3}} \frac{i p_{+}^{\prime \prime} \frac{\Delta_{\mathrm{pL}}}{\omega_{\mathrm{a}}}+p_{+}^{\prime}\left(1+i \frac{\gamma_{2}}{\omega_{\mathrm{a}}}\right)}{\frac{D_{\mathrm{L}}}{\omega_{\mathrm{a}}^{3}}},
$$

where $p_{+}^{\prime}\left(p_{+}^{\prime \prime}\right)$ is the real (imaginary) part of $p_{+}$, i.e.

$$
\begin{aligned}
& p_{+}^{\prime}=\frac{p_{+}+p_{+}^{*}}{2}, \\
& p_{+}^{\prime \prime}=\frac{p_{+}-p_{+}^{*}}{2 i} .
\end{aligned}
$$

To second order in $g_{\mathrm{a}}$ the term $\Lambda_{1}$ [see Eq. (A39)] can be calculated by evaluating the fixed point value of $p_{+}$ to zeroth order in $g_{\mathrm{a}}$, which is done by solving the set of equations $\Theta_{\mathrm{a}}=0, \Theta_{+}=0$ and $\Theta_{z}=0$ for the case $g_{\mathrm{a}}=0$. The steady state values of the variables $a, p_{+}$ and $p_{z}$ are found to be given by $a_{0}=0$,

$$
\begin{gathered}
p_{+0}=\frac{\frac{\omega_{1}}{\gamma_{2}}\left(-\frac{\Delta_{\mathrm{pL}}}{\gamma_{2}}-i\right) p_{0}}{1+\frac{\Delta_{\mathrm{pL}}^{2}}{\gamma_{2}^{2}}+\frac{4 \omega_{1}^{2}}{\gamma_{1} \gamma_{2}}}, \\
p_{z 0}=\frac{\left(1+\frac{\Delta_{\mathrm{pL}}^{2}}{\gamma_{2}^{2}}\right) p_{0}}{1+\frac{\Delta_{\mathrm{pL}}^{2}}{\gamma_{2}^{2}}+\frac{4 \omega_{1}^{2}}{\gamma_{1} \gamma_{2}}},
\end{gathered}
$$

respectively. For the case where $\gamma_{1}, \gamma_{2} \ll \omega_{\mathrm{a}}$ Eqs. (A37) and (A38) become

$$
\frac{\omega_{\mathrm{dR}}}{\omega_{\mathrm{a}}}=\sqrt{1+\frac{2 \gamma_{2}}{\gamma_{1}}\left(1-\frac{2 \omega_{1}^{2}}{\omega_{\mathrm{a}}^{2}}\right)},
$$

and

$$
\frac{\omega_{\mathrm{dI}}}{\omega_{\mathrm{a}}}=\sqrt{1-\frac{4 \omega_{1}^{2}}{\omega_{\mathrm{a}}^{2}}} .
$$

With the help of Eqs. (A36), (A39), (A44) and A45 one obtains for this case

$$
\frac{\Lambda_{1}}{\omega_{\mathrm{a}}}=-\frac{\frac{\frac{8 g_{\mathrm{a}}^{2} \omega_{1}^{2}}{\omega_{\mathrm{a}}^{3} \gamma_{2}} \frac{\Delta_{\mathrm{pL}}}{\gamma_{2}}\left(1+\frac{2 i \gamma_{2}}{\omega_{\mathrm{a}}}\right)}{1+\frac{\Delta_{\mathrm{pL}}^{2}}{\gamma_{2}^{2}}+\frac{4 \omega_{1}^{2}}{\gamma_{1} \gamma_{2}}} p_{0}}{\frac{\gamma_{1}}{\omega_{\mathrm{a}}}\left(\frac{\omega_{\mathrm{R}}^{2}+\eta \omega_{\mathrm{a}}^{2}}{\omega_{\mathrm{a}}^{2}}-1\right)-i\left(\frac{\omega_{\mathrm{R}}^{2}}{\omega_{\mathrm{a}}^{2}}-1\right)},
$$

where

$$
\eta=\frac{2 \gamma_{2}}{\gamma_{1}}\left[\left(1-\frac{\gamma_{1}}{\gamma_{2}}\right) \frac{2 \omega_{1}^{2}}{\omega_{\mathrm{a}}^{2}}-1\right]
$$

and where $\omega_{\mathrm{R}}=\sqrt{4 \omega_{1}^{2}+\Delta_{\mathrm{pL}}^{2}}$ is the Rabi frequency of the driven spins.

\section{Appendix B: Intermode coupling}

In general, Eq. A26) can be employed for calculating the eigenvalue of a cavity mode that is weakly coupled 
to any given ancila system. In the previous section the ancila system under consideration was an ensemble of driven spins, whereas in the current section the ancila system is taken to be the driven spiral mode. In general, the second order term $-g^{2}\left(V R\left(\omega_{\mathrm{a}}\right) V\right)_{11}$ in Eq. (A26) can be calculated by evaluating the steady state response of the ancilla system to small monochromatic oscillations of the cavity mode at its own resonance frequency. Substituting the steady state solution into the equation of motion of the cavity mode gives its eigenvalue. This approach will be employed in this section.

The Hamiltonian of the two-mode cavity closed system is taken to be given by

$$
\begin{aligned}
\hbar^{-1} \mathcal{H}_{\mathrm{ab}} & =\omega_{\mathrm{a}}\left(A_{\mathrm{a}}^{\dagger} A_{\mathrm{a}}+\frac{1}{2}\right)+\omega_{\mathrm{b}}\left(A_{\mathrm{b}}^{\dagger} A_{\mathrm{b}}+\frac{1}{2}\right) \\
& +K\left(A_{\mathrm{a}}+A_{\mathrm{a}}^{\dagger}\right)\left(A_{\mathrm{b}}+A_{\mathrm{b}}^{\dagger}\right)^{2},
\end{aligned}
$$

where $\omega_{\mathrm{a}}$ and $A_{\mathrm{a}}\left(\omega_{\mathrm{b}}\right.$ and $\left.A_{\mathrm{b}}\right)$ are the angular frequency and the annihilation operator, respectively, of the omega (spiral) resonator, and $K$ is the intermode coupling coefficient. The Heisenberg equations of motion are given by [see Eq. (A2)]

$$
\begin{array}{r}
\frac{\mathrm{d} A_{\mathrm{a}}}{\mathrm{d} t}+i \omega_{\mathrm{a}} A_{\mathrm{a}}+i K\left(A_{\mathrm{b}}+A_{\mathrm{b}}^{\dagger}\right)^{2}=0, \\
\frac{\mathrm{d} A_{\mathrm{b}}}{\mathrm{d} t}+i \omega_{\mathrm{b}} A_{\mathrm{b}}+2 i K\left(A_{\mathrm{a}}+A_{\mathrm{a}}^{\dagger}\right)\left(A_{\mathrm{b}}+A_{\mathrm{b}}^{\dagger}\right)=0 .
\end{array}
$$

Adding damping and driving leads to

$$
\frac{\mathrm{d} A_{\mathrm{a}}}{\mathrm{d} t}+\left(i \omega_{\mathrm{a}}+\gamma_{\mathrm{a}}\right) A_{\mathrm{a}}+i K\left(A_{\mathrm{b}}+A_{\mathrm{b}}^{\dagger}\right)^{2}=F_{\mathrm{a}},
$$

and

$$
\begin{aligned}
& \frac{\mathrm{d} A_{\mathrm{b}}}{\mathrm{d} t}+\left(i \omega_{\mathrm{b}}+\gamma_{\mathrm{b}}\right) A_{\mathrm{b}}+2 i K\left(A_{\mathrm{a}}+A_{\mathrm{a}}^{\dagger}\right)\left(A_{\mathrm{b}}+A_{\mathrm{b}}^{\dagger}\right) \\
& =F_{\mathrm{bf}} e^{-i\left(\omega_{\mathrm{b}}+\omega_{\mathrm{D}}\right) t}+F_{\mathrm{b}}
\end{aligned}
$$

where both noise terms $F_{\mathrm{a}}$ and $F_{\mathrm{b}}$ have a vanishing expectation value. Averaging yields

$$
\frac{\mathrm{d} \mathcal{A}_{\mathrm{a}}}{\mathrm{d} t}+\left(i \omega_{\mathrm{a}}+\gamma_{\mathrm{a}}\right) \mathcal{A}_{\mathrm{a}}+i K\left(\mathcal{A}_{\mathrm{b}}+\mathcal{A}_{\mathrm{b}}^{*}\right)^{2}=0
$$

and

$$
\begin{aligned}
& \frac{\mathrm{d} \mathcal{A}_{\mathrm{b}}}{\mathrm{d} t}+\left(i \omega_{\mathrm{b}}+\gamma_{\mathrm{b}}\right) \mathcal{A}_{\mathrm{b}}+S_{\mathrm{b} 1}+S_{\mathrm{b} 2} \\
& =F_{\mathrm{bf}} e^{-i\left(\omega_{\mathrm{b}}+\omega_{\mathrm{D}}\right) t} .
\end{aligned}
$$

where

$$
\begin{aligned}
& \left\langle A_{\mathrm{a}}\right\rangle=\mathcal{A}_{\mathrm{a}}=a_{\mathrm{a}} e^{-i \omega_{\mathrm{a}} t}, \\
& \left\langle A_{\mathrm{b}}\right\rangle=\mathcal{A}_{\mathrm{b}}=a_{\mathrm{b}} e^{-i \omega_{\mathrm{b}} t},
\end{aligned}
$$

and where

$$
\begin{aligned}
& S_{\mathrm{b} 1}=2 i K\left(\mathcal{A}_{\mathrm{a}}+\mathcal{A}_{\mathrm{a}}^{*}\right) \mathcal{A}_{\mathrm{b}}, \\
& S_{\mathrm{b} 2}=2 i K\left(\mathcal{A}_{\mathrm{a}}+\mathcal{A}_{\mathrm{a}}^{*}\right) \mathcal{A}_{\mathrm{b}}^{*} .
\end{aligned}
$$

In the subsections below the effect of the terms $S_{\mathrm{b} 1}$ and $S_{\mathrm{b} 2}$ is separately evaluated.

\section{The effect of the $S_{\mathrm{b} 1}$ term}

When the term $S_{\mathrm{b} 2}$ is disregarded Eq. (B7) becomes

$$
\frac{\mathrm{d} C_{\mathrm{b}}}{\mathrm{d} t}+\left(i \Omega_{\mathrm{b}}+\gamma_{\mathrm{b}}\right) C_{\mathrm{b}}=F_{\mathrm{bf}},
$$

where

$$
\Omega_{\mathrm{b}}=-\omega_{\mathrm{D}}+2 K\left(\mathcal{A}_{\mathrm{a}}+\mathcal{A}_{\mathrm{a}}^{*}\right)
$$

and where

$$
\mathcal{A}_{\mathrm{b}}=C_{\mathrm{b}} e^{-i\left(\omega_{\mathrm{b}}+\omega_{\mathrm{D}}\right) t} .
$$

By employing the notation

$$
C_{\mathrm{b}}=C_{\mathrm{b} 0}+c_{\mathrm{b}},
$$

where

$$
C_{\mathrm{b} 0}=\frac{F_{\mathrm{bf}}}{-i \omega_{\mathrm{D}}+\gamma_{\mathrm{b}}}
$$

one obtains in the limit of small $K$

$$
\frac{\mathrm{d} c_{\mathrm{b}}}{\mathrm{d} t}+\left(-i \omega_{\mathrm{D}}+\gamma_{\mathrm{b}}\right) c_{\mathrm{b}}=-2 i K\left(\mathcal{A}_{\mathrm{a}}+\mathcal{A}_{\mathrm{a}}^{*}\right) C_{\mathrm{b} 0}
$$

Let $\mathcal{A}_{\mathrm{a}}=a_{\mathrm{a}} e^{-i \omega_{\mathrm{a}} t}[$ see Eq. (B8) $]$, and assume that $a_{\mathrm{a}}$ is constant. The steady state solution reads

$$
c_{\mathrm{b}}=\frac{2 i K C_{\mathrm{b} 0} \mathcal{A}_{\mathrm{a}}}{i\left(\omega_{\mathrm{D}}+\omega_{\mathrm{a}}\right)-\gamma_{\mathrm{b}}}+\frac{2 i K C_{\mathrm{b} 0} \mathcal{A}_{\mathrm{a}}^{*}}{i\left(\omega_{\mathrm{D}}-\omega_{\mathrm{a}}\right)-\gamma_{\mathrm{b}}} .
$$

When only terms proportional to $\mathcal{A}_{\mathrm{a}}$ are kept, one finds the coupling term in Eq. (B6) can be expressed as

$$
\begin{aligned}
& i K\left(\mathcal{A}_{\mathrm{b}}+\mathcal{A}_{\mathrm{b}}^{*}\right)^{2} \\
& \simeq \frac{4 i K^{2}\left|C_{\mathrm{b} 0}\right|^{2} \omega_{\mathrm{D}} \mathcal{A}_{\mathrm{a}}}{\left[i\left(\omega_{\mathrm{a}}-\omega_{\mathrm{D}}\right)-\gamma_{\mathrm{b}}\right]\left[i\left(\omega_{\mathrm{a}}+\omega_{\mathrm{D}}\right)-\gamma_{\mathrm{b}}\right]} .
\end{aligned}
$$

\section{The effect of the $S_{\mathrm{b} 2}$ term}

For this case the term $S_{\mathrm{b} 1}$ in Eq. (B7) is disregarded. Furthermore, the counter rotating term proportional to $\mathcal{A}_{\mathrm{a}}^{*} \mathcal{A}_{\mathrm{b}}^{*}$ is disregarded as well [see Eq. (B11)]. For this case Eq. (B7) becomes

$$
\frac{\mathrm{d} a_{\mathrm{b}}}{\mathrm{d} t}+\gamma_{\mathrm{b}} a_{\mathrm{b}}+2 i K a_{\mathrm{a}} a_{\mathrm{b}}^{*} e^{i \omega_{\mathrm{s}} t}=F_{\mathrm{bf}} e^{-i \omega_{\mathrm{D}} t},
$$


where

$$
\omega_{\mathrm{s}}=2 \omega_{\mathrm{b}}-\omega_{\mathrm{a}} .
$$

Consider a solution of Eq. (B20) having the form [26]

$$
a_{\mathrm{b}}=\alpha e^{-i \omega_{\mathrm{D}} t}+\beta e^{i\left(\omega_{\mathrm{s}}+\omega_{\mathrm{D}}\right) t} .
$$

Substituting the solution into Eq. (B-B20 and assuming that $\alpha, \beta$ and $a_{\mathrm{a}}$ are all constants lead to

$$
\left(-i \omega_{\mathrm{D}}+\gamma_{\mathrm{b}}\right) \alpha+2 i K a_{\mathrm{a}} \beta^{*}=F_{\mathrm{bf}},
$$

and

$$
\left[i\left(\omega_{\mathrm{s}}+\omega_{\mathrm{D}}\right)+\gamma_{\mathrm{b}}\right] \beta+2 i K a_{\mathrm{a}} \alpha^{*}=0,
$$

thus

$$
\alpha=\frac{F_{\mathrm{bf}}}{-i \omega_{\mathrm{D}}+\gamma_{\mathrm{b}}-\frac{4 K^{2}\left|a_{\mathrm{a}}\right|^{2}}{-i\left(\omega_{\mathrm{s}}+\omega_{\mathrm{D}}\right)+\gamma_{\mathrm{b}}}},
$$

and

$$
\beta=\frac{-2 i K a_{\mathrm{a}} \alpha^{*}}{i\left(\omega_{\mathrm{s}}+\omega_{\mathrm{D}}\right)+\gamma_{\mathrm{b}}} .
$$

The steady state solution (B22) can be used to express the coupling term $i K\left(\mathcal{A}_{\mathrm{b}}+\mathcal{A}_{\mathrm{b}}^{*}\right)^{2}$ in Eq. (B6) in terms of $\mathcal{A}_{\mathrm{a}}$. To that end $\mathcal{A}_{\mathrm{b}}$ is expressed as [see Eqs. (B8),
(B22) and (B26)]

$$
\begin{aligned}
\mathcal{A}_{\mathrm{b}} & =\alpha e^{-i\left(\omega_{\mathrm{b}}+\omega_{\mathrm{D}}\right) t}+\beta e^{i\left(\omega_{\mathrm{b}}+\omega_{\mathrm{D}}-\omega_{\mathrm{a}}\right) t} \\
& =\alpha e^{-i\left(\omega_{\mathrm{b}}+\omega_{\mathrm{D}}\right) t}-\frac{2 i K \alpha^{*} e^{i\left(\omega_{\mathrm{b}}+\omega_{\mathrm{D}}\right) t}}{i\left(\omega_{\mathrm{s}}+\omega_{\mathrm{D}}\right)+\gamma_{\mathrm{b}}} \mathcal{A}_{\mathrm{a}} .
\end{aligned}
$$

When only terms proportional to $\mathcal{A}_{\mathrm{a}}$ are kept, the following approximation is employed [see Eq. B25]

$$
\alpha \simeq \frac{F_{\mathrm{bf}}}{-i \omega_{\mathrm{D}}+\gamma_{\mathrm{b}}},
$$

and it is assumed that $\left|\omega_{\mathrm{D}}\right| \ll\left|\omega_{\mathrm{s}}\right|$ for evaluating $\beta$ [see Eq. (B26)] the coupling term in Eq. (Bי) becomes

$$
\begin{aligned}
i K\left(\mathcal{A}_{\mathrm{b}}+\mathcal{A}_{\mathrm{b}}^{*}\right)^{2} & \simeq-\frac{4 K^{2}|\alpha|^{2} \mathcal{A}_{\mathrm{a}}}{i\left(\omega_{\mathrm{s}}+\omega_{\mathrm{D}}\right)+\gamma_{\mathrm{b}}} \\
& \simeq-\frac{4 K^{2}\left|F_{\mathrm{bf}}\right|^{2} \mathcal{A}_{\mathrm{a}}}{\left(i \omega_{\mathrm{s}}+\gamma_{\mathrm{b}}\right)\left(\omega_{\mathrm{D}}^{2}+\gamma_{\mathrm{b}}^{2}\right)} .
\end{aligned}
$$

When $\gamma_{\mathrm{b}} \ll \omega_{\mathrm{s}}$ one has

$$
i K\left(\mathcal{A}_{\mathrm{b}}+\mathcal{A}_{\mathrm{b}}^{*}\right)^{2} \simeq \frac{4 K^{2}\left|F_{\mathrm{bf}}\right|^{2}\left(i \omega_{\mathrm{s}}-\gamma_{\mathrm{b}}\right) \mathcal{A}_{\mathrm{a}}}{\omega_{\mathrm{s}}^{2}\left(\omega_{\mathrm{D}}^{2}+\gamma_{\mathrm{b}}^{2}\right)} .
$$

[1] Serge Haroche and Daniel Kleppner, "Cavity quantum electrodynamics," Phys. Today, vol. 42, no. 1, pp. 24-30, 1989.

[2] Bruce W Shore and Peter L Knight, "The jaynescummings model," Journal of Modern Optics, vol. 40, no. 7, pp. 1195-1238, 1993.

[3] Eyal Buks, Chunqing Deng, Jean-Luc F. X. Orgazzi, Martin Otto, and Adrian Lupascu, "Superharmonic resonances in a strongly coupled cavity-atom system," Phys. Rev. A, vol. 94, pp. 033807, Sep 2016.

[4] S Ates, SM Ulrich, A Ulhaq, S Reitzenstein, A Löfller, S Höfling, A Forchel, and P Michler, "Non-resonant dot-cavity coupling and its potential for resonant singlequantum-dot spectroscopy," Nature Photonics, vol. 3, no. 12 , pp. 724-728, 2009.

[5] A. Ghirri, C. Bonizzoni, F. Troiani, N. Buccheri, L. Beverina, A. Cassinese, and M. Affronte, "Coherently coupling distinct spin ensembles through a high- $T_{c}$ superconducting resonator," Phys. Rev. A, vol. 93, pp. 063855, Jun 2016.

[6] Yung Szen Yap, Hiroshi Yamamoto, Yutaka Tabuchi, Makoto Negoro, Akinori Kagawa, and Masahiro Kitagawa, "Strongly driven electron spins using a $\mathrm{k} \mathrm{u}$ band stripline electron paramagnetic resonance resonator," Journal of Magnetic Resonance, vol. 232, pp. 62-67, 2013.

[7] Alberto Ghirri, Claudio Bonizzoni, Dario Gerace, Samuele Sanna, Antonio Cassinese, and Marco Affronte, "Yba2cu3o7 microwave resonators for strong collective coupling with spin ensembles," Applied Physics Letters, vol. 106, no. 18, pp. 184101, 2015.

[8] Tomás Ramos, Vivishek Sudhir, Kai Stannigel, Peter Zoller, and Tobias J Kippenberg, "Nonlinear quantum optomechanics via individual intrinsic two-level defects," Physical review letters, vol. 110, no. 19, pp. 193602, 2013.

[9] Lior Ella and Eyal Buks, "Hopf bifurcation in a flux qubit coupled to a nanomechanical oscillator," arXiv:1210.6902, 2012.

[10] M Grajcar, SHW Van der Ploeg, A Izmalkov, E Ilichev, H-G Meyer, A Fedorov, A Shnirman, and Gerd Schön, "Sisyphus cooling and amplification by a superconducting qubit," Nature physics, vol. 4, no. 8, pp. 612-616, 2008.

[11] JM de Voogd, JJT Wagenaar, and TH Oosterkamp, "Dissipation and resonance frequency shift of a resonator magnetically coupled to a semiclassical spin," arXiv:1508.07972, 2015.

[12] R Glenn and ME Raikh, "Rabi-vibronic resonance with large number of vibrational quanta," Physical Review B, vol. 84, no. 19, pp. 195454, 2011.

[13] Y. Twig, E. Suhovoy, and A. Blank, "Sensitive surface loop-gap microresonators for electron spin resonance.," Rev. Sci. Instrum., vol. 81, no. 10, pp. 104703, 2010.

[14] N Maleeva, M Fistul, A Averkin, A Karpov, and AV Ustinov, "Electrodynamics of a spiral resonator as a suitable magnetic component of metamaterials," Proceedings of Metamaterials, pp. 474-477, 2013.

[15] N Maleeva, MV Fistul, A Karpov, AP Zhuravel, 
A Averkin, P Jung, and AV Ustinov, "Electrodynamics of a ring-shaped spiral resonator," Journal of Applied Physics, vol. 115, no. 6, pp. 064910, 2014.

[16] DE Kaplan, ME Browne, and JA Cowen, "Pulsed x-band epr spectrometer," Review of Scientific Instruments, vol. 32, no. 11, pp. 1182-1186, 1961.

[17] JP Lloyd and GE Pake, "Spin-lattice relaxation and the residual width of highly exchange-narrowed paramagnetic resonances," Physical Review, vol. 92, no. 6, pp. 1576, 1953.

[18] DI Schuster, AP Sears, E Ginossar, L DiCarlo, L Frunzio, JJL Morton, $\mathrm{H}$ Wu, GAD Briggs, BB Buckley, DD Awschalom, et al., "High-cooperativity coupling of electron-spin ensembles to superconducting cavities," Physical review letters, vol. 105, no. 14, pp. 140501, 2010.

[19] Markus Aspelmeyer, Tobias J Kippenberg, and Florian Marquardt, "Cavity optomechanics," Reviews of Modern Physics, vol. 86, no. 4, pp. 1391, 2014.

[20] AA Houck, JA Schreier, BR Johnson, JM Chow, Jens Koch, JM Gambetta, DI Schuster, L Frunzio, MH Devoret, SM Girvin, et al., "Controlling the spontaneous emission of a superconducting transmon qubit," Physical review letters, vol. 101, no. 8, pp. 080502, 2008.

[21] Alexandre Blais, Ren-Shou Huang, Andreas Wallraff, SM Girvin, and R Jun Schoelkopf, "Cavity quantum electrodynamics for superconducting electrical circuits: An architecture for quantum computation," Physical Review $A$, vol. 69, no. 6, pp. 062320, 2004.
[22] Jens Koch, M Yu Terri, Jay Gambetta, Andrew A Houck, DI Schuster, J Majer, Alexandre Blais, Michel H Devoret, Steven M Girvin, and Robert J Schoelkopf, "Chargeinsensitive qubit design derived from the cooper pair box," Physical Review A, vol. 76, no. 4, pp. 042319, 2007.

[23] A. Wallraff, D. I. Schuster, A. Blais, L. Frunzio, R.-S. Huang, J. Majer, S. Kumar, S. M. Girvin, and R. J. Schoelkopf, "Strong coupling of a single photon to a superconducting qubit using circuit quantum electrodynamics," Nature, vol. 431, pp. 162-167, 2004.

[24] Thomas Niemczyk, F Deppe, H Huebl, EP Menzel, F Hocke, MJ Schwarz, JJ Garcia-Ripoll, D Zueco, T Hümmer, E Solano, et al., "Circuit quantum electrodynamics in the ultrastrong-coupling regime," Nature Physics, vol. 6, no. 10, pp. 772-776, 2010.

[25] Pol Forn-Díaz, J Lisenfeld, David Marcos, Juan José García-Ripoll, Enrique Solano, CJPM Harmans, and JE Mooij, "Observation of the Bloch-Siegert shift in a qubit-oscillator system in the ultrastrong coupling regime," Physical Review Letters, vol. 105, no. 23, pp. 237001, 2010.

[26] R Lifshitz and M.C. Cross, "Nonlinear dynamics of nanomechanical and micromechanical resonators," in Reviews of nonlinear dynamics and complexity, Heinz Georg Schuster, Ed., vol. 1, pp. 1-48. Wiley-VCH, 2008. 